$\square$ TECHNISCHE UNIVERSITÄT

- KAISERSLAUTERN

\title{
SCHRIFTEN ZUR
}

\section{FUNKTIONALANALYSIS UND GEOMATHEMATIK}

T. Fehlinger, W. Freeden, S. Gramsch,

C. Mayer, D. Michel, M. Schreiner

Local Modelling of Sea Surface Topography

from (Geostrophic) Ocean Flow

Bericht 31 - Januar 2007 


\title{
Local Modelling of Sea Surface Topography from (Geostrophic) Ocean Flow
}

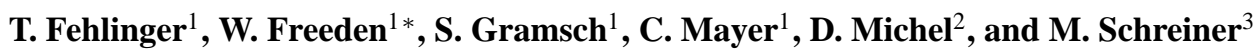 \\ ${ }^{1}$ University of Kaiserslautern \\ Geomathematics Group \\ 67663 Kaiserslautern \\ P.O. Box 3049 \\ Germany \\ 2 Proudman Oceanographic Laboratory \\ 6 Brownlow Street \\ Liverpool L3 5DA \\ UK \\ ${ }^{3}$ University of Buchs NTB \\ Laboratory for Industrial Mathematics \\ Werdenbergstrasse 4 \\ CH-9471 Buchs \\ Switzerland
}

Key words Geostrophic flow, locally supported (Green's) vector wavelets, local approximation of sea surface topography.

MSC (2000) 34B27 65T60 86A05

\begin{abstract}
This paper deals with the problem of determining the sea surface topography from geostrophic flow of ocean currents on local domains of the spherical Earth. In mathematical context the problem amounts to the solution of a spherical differential equation relating the surface curl gradient of a scalar field (sea surface topography) to a surface divergence-free vector field (geostrophic ocean flow).

At first, a continuous solution theory is presented in the framework of an integral formula involving Green's function of the spherical Beltrami operator. Different criteria derived from spherical vector analysis are given to investigate uniqueness. Second, for practical applications Green's function is replaced by a regularized counterpart. The solution is obtained by a convolution of the flow field with a scaled version of the regularized Green function.

Calculating locally without boundary correction would lead to errors near the boundary. To avoid these Gibbs phenomenona we additionally consider the boundary integral of the corresponding region on the sphere which occurs in the integral formula of the solution. For reasons of simplicity we discuss a spherical cap first, that means we consider a continuously differentiable (regular) boundary curve. In a second step we concentrate on a more complicated domain with a non continuously differentiable boundary curve, namely a rectangular region. It will turn out that the boundary integral provides a major part for stabilizing and reconstructing the approximation of the solution in our multiscale procedure.
\end{abstract}

\section{Introduction}

As a crucial factor for weather and climate, the oceans are of immediate importance. By modelling oceanic currents on the sphere (and in a first approximation the regions under consideration may be assumed to be part of a spherically shaped Earth), we gain a better understanding of meteorological processes. The point of departure for our intention to determine the sea surface topography is the geostrophic flow equation derived in conventional form from the basic hydrodynamic equation (see, e.g., $[1,5,11,12]$ ). As a scalar field on the spherical Earth, the sea surface topography $H$ consists of two ingredients. On the one hand, on an Earth at rest, the water masses would align along the geoid (given as the deviation from

\footnotetext{
* Corresponding author, e-mail: freeden@mathematik.uni-kl.de, Phone: +49631205 3867, Fax: +49631 2054736
} 
the standard reference ellipsoid used in physical geodesy (see [7, 8])). On the other hand, satellite measurements provide altimetric data of the actual sea surface height which in marine geodesy are also used (in relation to the standard reference ellipsoid). The difference between these quantities can, of course, be considered as the actual sea surface topography. In other words, the sea surface topography $H$ is defined as the deviation of the ocean surface from the geoidal surface which is here assumed to be due to the geostrophic component of the ocean currents. The surface geostrophic velocity of the ocean currents then reads as follows (see $[1,5,11,12])$

$$
L_{\xi}^{*} H(\xi)=h(\xi), \quad \xi \in \Omega^{o c}
$$

where $\Omega^{o c}$ is the subset of the Earth's sphere $\Omega \subset \mathbb{R}^{3}$ being covered by oceans (for simplicity, we associate $\Omega$ with the unit sphere in $\mathbb{R}^{3}$ ), $L_{\xi}^{*}$ is the surface curl gradient acting on $\xi \in \Omega^{o c}$, defined by $L_{\xi}^{*}=\xi \wedge \nabla_{\xi}^{*}$ with $\nabla^{*}$ the surface gradient. Further, $h(\xi)$ is the product of three factors, namely (i) the quotient between the mean equatorial Earth's radius $R$ and the acceleration of gravity $g(\xi)$ at $\xi$, (ii) the Coriolis force coefficient $c(\xi)=2|\omega|\left(\varepsilon^{3} \cdot \xi\right)$ (with $|\omega|$ the angular velocity of the Earth), and (iii) the velocity field $v(\xi)$ at $\xi$. In detail,

$$
h(\xi)=\frac{2 R}{g(\xi)}|\omega|\left(\varepsilon^{3} \cdot \xi\right) v(\xi), \quad \xi \in \Omega^{o c} .
$$

Clearly, for all $\xi \in \Omega^{o c}$, the geostrophic flow $h$ given by (1.1) is perpendicular to the tangential surface gradient $\nabla^{*} H$ of the sea surface topography on $\Omega^{o c}$. This is a remarkable feature of the geostrophic velocity field. The currents flow along and not across the lines of constant sea surface topography.

In this paper we are concerned with the so called Topography Problem of geostrophic ocean circulation in a normal region $\Gamma \subset \Omega^{o c}$ : Let the vectorial velocity field $h$ be known for a finite subset of points $\left\{\eta_{1}, \ldots, \eta_{N}\right\}$ on $\Gamma$, and the scalar sea surface topography $H$ be known for a set $\left\{\tilde{\eta}_{1}, \ldots, \tilde{\eta}_{\tilde{N}}\right\}$ on the boundary $\partial \Gamma$ of $\Gamma$. Find an approximation of $H$ from the discrete data $\left\{\left(\eta_{i}, h\left(\eta_{i}\right)\right\}_{i=1, \ldots, N}\right.$ and $\left\{\left(\tilde{\eta}_{i}, H\left(\tilde{\eta}_{i}\right)\right\}_{i=1, \ldots, \tilde{N}}\right.$ on the whole domain $\bar{\Gamma}=\Gamma \cup \partial \Gamma$.

In oceanography the sea surface topography is conventionally represented (on the whole Earth's surface) on the sphere $\Omega$ by a conventional Fourier (orthogonal) expansion in terms of spherical harmonics $Y_{n, j}$ (see, e.g., [10]) thereby assuming $\int_{\Omega} H(\xi) Y_{0,1}(\xi) d \omega(\xi)=\int_{\Omega^{\circ c}} H(\xi) Y_{0,1}(\xi) d \omega(\xi)=0$ ( $d \omega$ denotes the surface element).This approach ignores the local formulation of the problem (1.1) for the oceanic part $\overline{\Omega^{o c}}$ of the (spherical) Earth's surface. Instead it leads to an orthogonal series expansion of the velocity field in terms of surface divergence free vector spherical harmonics $L^{*} Y_{n, j}$ on the whole (spherical) Earth. But obviously, these vector types of polynomial functions are far from being suitable for purposes of approximation on the oceanic area $\Omega^{o c}$. First, the constituting ingredients of spherical harmonics show certain phenomena of (global) periodicity at least when the classical basis system involving associated Legendre functions is used. In consequence, the assumptions on continents influence the approximation on oceans. Second, boundary effects along the coast lines like the Gibbs phenomenon are not avoidable by use of spherical harmonics, i.e., by use of non-space-localizing polynomials. So it is really necessary for geoscientific practice to develop on a geostrophic theory of ocean circulation in restriction to the oceanic parts $\Omega^{o c}$ on the Earth's surface thereby using specific space-localizing (trial) kernel vector functions for purposes of approximation on $\Omega^{o c}$. These requirements will be investigated in the approach presented below.

\section{Preliminaries}

Throughout this paper we need a number of differential operators on the unit sphere $\Omega \subset \mathbb{R}^{3}$ which are listed in Table 1 (see, e.g., [4]).

Table 1 Differential operators

\begin{tabular}{ll}
\hline Symbol & Differential Operator \\
\hline$\nabla_{x}$ & Gradient at $x$ \\
$\Delta_{x}=\nabla_{x} \cdot \nabla_{x}$ & Laplace operator at $x$ \\
$\nabla_{\xi}^{*}$ & Surface gradient on the unit sphere $\Omega$ at $\xi$ \\
$L_{\xi}^{*}=\xi \wedge \nabla_{\xi}^{*}$ & Surface curl gradient on the unit sphere $\Omega$ at $\xi$ \\
$\Delta_{\xi}^{*}=\nabla_{\xi}^{*} \cdot \nabla_{\xi}^{*}=L_{\xi}^{*} \cdot L_{\xi}^{*}$ & Beltrami operator on the unit sphere $\Omega$ at $\xi$ \\
$\nabla_{\xi}^{*}$ & Surface divergence on the unit sphere $\Omega$ at $\xi$ \\
$L_{\xi}^{*}$ & Surface curl on the unit sphere $\Omega$ at $\xi$ \\
\hline
\end{tabular}


It should be noted that the operators $\nabla^{*}, L^{*}$, and $\Delta^{*}$ will be always used in a coordinate-free representation, thereby avoiding any kind of singularities at the poles. Moreover, following the nomenclature of [4] we denote by $\nabla^{*}$. the surface divergence on $\Omega$ and by $L^{*}$. the surface curl on $\Omega$. Clearly, $\Delta^{*}=L^{*} \cdot L^{*}=\nabla^{*} \cdot \nabla^{*}$. Note that the operators $\nabla^{*}, L^{*}, \Delta^{*}$ show special features in certain situations (for more details the reader is referred to [4]). For example, let $\eta \in \Omega$ be fixed. If $G$ is of class $C^{(1)}[-1,1]$ and $G^{\prime} \in C^{(0)}[-1,1]$ denotes its derivative, then we find

$$
\begin{aligned}
\nabla_{\xi}^{*} G(\xi \cdot \eta) & =G^{\prime}(\xi \cdot \eta)(\eta-(\eta \cdot \xi) \xi), \quad \xi \in \Omega, \\
L_{\xi}^{*} G(\xi \cdot \eta) & =G^{\prime}(\xi \cdot \eta)(\xi \wedge \eta), \quad \xi \in \Omega
\end{aligned}
$$

whereas for $G \in C^{(2)}[-1,1]$

$$
\Delta_{\xi}^{*} G(\xi \cdot \eta)=\left(L^{*} \cdot L^{*}\right) G(\xi \cdot \eta)=-2(\xi \cdot \eta) G^{\prime}(\xi \cdot \eta)+\left(1-(\xi \cdot \eta)^{2}\right) G^{\prime \prime}(\xi \cdot \eta), \quad \xi \in \Omega .
$$

\section{Green's Theorems on (Normal) Regions of the Sphere}

A standard method for solving boundary value problems corresponding to the Beltrami operator $\Delta^{*}$ is the theory of Green's functions. We first introduce the definition and the properties of Green's function with respect to the Beltrami operator $\Delta^{*}$ (see [3]). Then we prove the fundamental theorem for $L^{*}$ on normal regions on the sphere in order to solve differential problems involving the surface curl gradient (cf. [3, 6]).

Definition 3.1 The function $G\left(\Delta^{*} ; \cdot, \cdot\right):(\xi, \eta) \mapsto G\left(\Delta^{*} ; \xi, \eta\right), \xi, \eta \in \Omega$ with $-1 \leq \xi \cdot \eta<1$, is called Green's function on $\Omega$ with respect to the operator $\Delta^{*}$, if it satisfies the following properties:

1. (differential equation) For every point $\xi \in \Omega, \eta \mapsto G\left(\Delta^{*} ; \xi, \eta\right)$ is twice continuously differentiable on $\{\eta \in \Omega$ : $-1 \leq \xi \cdot \eta<1\}$, and we have

$$
\Delta_{\eta}^{*} G\left(\Delta^{*} ; \xi, \eta\right)=-\frac{1}{4 \pi}, \quad-1 \leq \xi \cdot \eta<1 .
$$

2. (characteristic singularity) For every $\xi \in \Omega$, the function

$$
\eta \mapsto G\left(\Delta^{*} ; \xi, \eta\right)-\frac{1}{4 \pi} \ln (1-\xi \cdot \eta)
$$

is continuously differentiable on $\Omega$.

3. (rotational symmetry) For all orthogonal transformations $\mathbf{t}$

$$
G\left(\Delta^{*} ; \mathbf{t} \xi, \mathbf{t} \eta\right)=G\left(\Delta^{*} ; \xi, \eta\right) .
$$

4. (normalization) For every $\xi \in \Omega$,

$$
\int_{\Omega} G\left(\Delta^{*} ; \xi, \eta\right) d \omega(\eta)=0
$$

Following [3] the uniqueness of Green's function with respect to $\Delta^{*}$ is guaranteed. In fact, the function

$$
G\left(\Delta^{*} ; \xi, \eta\right)=\frac{1}{4 \pi} \ln (1-\xi \cdot \eta)+\frac{1}{4 \pi}-\frac{1}{4 \pi} \ln 2, \quad-1 \leq \xi \cdot \eta<1,
$$

is an explicit representation of Green's function with respect to the Beltrami operator $\Delta^{*}$. In connection with (2.1) we obtain

$$
L_{\eta}^{*} G\left(\Delta^{*} ; \xi, \eta\right)=-\frac{1}{4 \pi} \frac{\eta \wedge \xi}{1-\xi \cdot \eta}, \quad-1 \leq \xi \cdot \eta<1 .
$$

Next, we explain some geometrical assumptions imposed on subsets of the unit sphere $\Omega \subset \mathbb{R}^{3}$.

Definition 3.2 A region, i.e., an open and connected set $\Gamma \subset \Omega$, is called normal if the surface theorem of Stokes

$$
\int_{\Gamma} L_{\xi}^{*} \cdot f(\xi) d \omega(\xi)=\int_{\partial \Gamma} \tau_{\xi} \cdot f(\xi) d \sigma(\xi)
$$

is valid for all continuously differentiable vector fields $f \in c^{(1)}(\Omega)$, where $\partial \Gamma$ is the boundary curve of $\Gamma$, $\tau$ is the unit surface field pointing tangential to $\partial \Gamma$ and $\sigma$ is the arc length along $\partial \Gamma$. A normal region $\Gamma \subset \Omega$ is called regular, if its boundary $\partial \Gamma$ has a continuously differentiable unit normal field $\nu: \partial \Gamma \rightarrow \mathbb{R}^{3}$ pointing outward of $\Gamma$, i.e., into $\Omega \backslash \bar{\Gamma}$. 
By choosing $f=F L^{*} Q, F \in C^{(1)}(\bar{\Gamma}), Q \in C^{(2)}(\bar{\Gamma})$ in the surface theorem of Stokes we get Green's surface identity for the operator $L^{*}$, viz.

$$
\int_{\Gamma}\left(L_{\eta}^{*} F(\eta) \cdot L_{\eta}^{*} Q(\eta)+F(\eta) \Delta_{\eta}^{*} Q(\eta)\right) d \omega(\eta)=\int_{\partial \Gamma} F(\eta) \tau_{\eta} \cdot L_{\eta}^{*} Q(\eta) d \sigma(\eta) .
$$

Let the function $F: \bar{\Gamma} \rightarrow \mathbb{R}$ be continuously differentiable and $\xi \in \bar{\Gamma}$ be fixed. Applying Green's surface identity to $F$ and Green's function $G\left(\Delta^{*} ; \xi, \cdot\right)$ on the region $\{\eta \in \partial \Gamma:|\xi-\eta| \geq \varepsilon\}$ we obtain for sufficiently small $\varepsilon>0$

$$
\begin{aligned}
& \int_{|\xi-\eta| \geq \varepsilon, \eta \in \Gamma}\left(L_{\eta}^{*} F(\eta) \cdot L_{\eta}^{*} G\left(\Delta^{*} ; \xi, \eta\right)+F(\eta) \Delta_{\eta}^{*} G\left(\Delta^{*} ; \xi, \eta\right)\right) d \omega(\eta) \\
& \quad=\int_{|\xi-\eta|=\varepsilon, \eta \in \Gamma} F(\eta) \tau_{\eta} \cdot L_{\eta}^{*} G\left(\Delta^{*} ; \xi, \eta\right) d \sigma(\eta)+\int_{|\xi-\eta| \geq \varepsilon, \eta \in \partial \Gamma} F(\eta) \tau_{\eta} \cdot L_{\eta}^{*} G\left(\Delta^{*} ; \xi, \eta\right) d \sigma(\eta),
\end{aligned}
$$

where $\sigma$ denotes the arc length along $\partial \Gamma$ and $\{\eta \in \Gamma:|\xi-\eta|=\varepsilon\}$, while $\tau$ is the unit surface vector tangential to $\{\eta \in \bar{\Gamma}:|\xi-\eta|=\varepsilon\}$ or $\{\eta \in \partial \Gamma:|\xi-\eta| \geq \varepsilon\}$, respectively. Using Property 1 of Definition 3.1

$$
\Delta_{\eta}^{*} G\left(\Delta^{*} ; \xi, \eta\right)=-\frac{1}{4 \pi}, \quad \eta \in \Gamma
$$

equation (3.3) can be rewritten as follows

$$
\begin{aligned}
& \int_{|\xi-\eta| \geq \varepsilon, \eta \in \Gamma} L_{\eta}^{*} F(\eta) \cdot L_{\eta}^{*} G\left(\Delta^{*} ; \xi, \eta\right) d \omega(\eta)-\frac{1}{4 \pi} \int_{|\xi-\eta| \geq \varepsilon, \eta \in \Gamma} F(\eta) d \omega(\eta) \\
& =\int_{|\xi-\eta|=\varepsilon, \eta \in \Gamma} F(\eta) \tau_{\eta} \cdot L_{\eta}^{*} G\left(\Delta^{*} ; \xi, \eta\right) d \sigma(\eta)+\int_{|\xi-\eta| \geq \varepsilon, \eta \in \partial \Gamma} F(\eta) \tau_{\eta} \cdot L_{\eta}^{*} G\left(\Delta^{*} ; \xi, \eta\right) d \sigma(\eta) .
\end{aligned}
$$

Next, we concentrate on the integral

$$
I_{\varepsilon}(\xi)=\int_{|\xi-\eta|=\varepsilon, \eta \in \Gamma} F(\eta) \tau_{\eta} \cdot L_{\eta}^{*} G\left(\Delta^{*} ; \xi, \eta\right) d \sigma(\eta) .
$$

For each point $\eta \in \Gamma$ with $|\xi-\eta|=\varepsilon$, we have

$$
\tau_{\eta}=\frac{\xi \wedge \eta}{\sqrt{1-(\xi \cdot \eta)^{2}}}
$$

Hence, we find with (3.1)

$$
I_{\varepsilon}(\xi)=-\frac{1}{4 \pi} \int_{|\xi-\eta|=\varepsilon, \eta \in \Gamma} F(\eta) \frac{\sqrt{1-(\xi \cdot \eta)^{2}}}{1-\xi \cdot \eta} d \sigma(\eta) .
$$

Letting $\varepsilon \rightarrow 0$ we obtain, in analogy to well-known results of potential theory (see e.g. [3, 9]),

$$
\lim _{\varepsilon \rightarrow 0} I_{\varepsilon}(\xi)=-\frac{\alpha(\xi)}{2 \pi} F(\xi)
$$

where $\alpha(\xi)$ is the solid angel subtended at $\xi \in \bar{\Gamma}$. This finally leads to the following integral formula.

Theorem 3.3 (Fundamental Theorem for $L^{*}$ on Normal Regions) Let $\Gamma$ be a normal region with boundary $\partial \Gamma$. Suppose that $F$ is a continuously differentiable function on $\bar{\Gamma}$, i.e., $F \in C^{(1)}(\bar{\Gamma})$. Then, for every point $\xi \in \Omega$, we have

$$
\frac{\alpha(\xi)}{2 \pi} F(\xi)=\frac{1}{4 \pi} \int_{\Gamma} F(\eta) d \omega(\eta)-\int_{\Gamma} L_{\eta}^{*} F(\eta) \cdot L_{\eta}^{*} G\left(\Delta^{*} ; \xi, \eta\right) d \omega(\eta)+\int_{\partial \Gamma} F(\eta) \tau_{\eta} \cdot L_{\eta}^{*} G\left(\Delta^{*} ; \xi, \eta\right) d \sigma(\eta)
$$

Setting, particularly, $F=1$ on $\bar{\Gamma}$ we immediately get from Theorem 3.3

$$
\alpha(\xi)=\frac{\|\Gamma\|}{2}+2 \pi \int_{\partial \Gamma} \tau_{\eta} \cdot L_{\eta}^{*} G\left(\Delta^{*} ; \xi, \eta\right) d \sigma(\eta), \quad\|\Gamma\|=\int_{\Gamma} d \omega .
$$

Clearly, in case of a regular region, $\alpha(\xi)=2 \pi$ for all $\xi \in \Gamma$ and $\alpha(\xi)=\pi$ for all $\xi \in \partial \Gamma$. Furthermore, for the whole sphere $\Omega$ we have the following result (cf. [3, 4]).

Corollary 3.4 (Fundamental Theorem for $L^{*}$ on $\Omega$ ) Suppose that $F$ is of class $C^{(1)}(\Omega)$. Then, for every $\xi \in \Omega$,

$$
F(\xi)=\frac{1}{4 \pi} \int_{\Omega} F(\eta) d \omega(\eta)-\int_{\Omega} L_{\eta}^{*} F(\eta) \cdot L_{\eta}^{*} G\left(\Delta^{*} ; \xi, \eta\right) d \omega(\eta)
$$




\section{Potential and Stream Functions}

Let us consider a continuous spherical vector field $f$ of class $c^{(0)}(\Omega)$. For all $\xi \in \Omega$ we call $\xi \rightarrow f_{\text {nor }}(\xi)=(f(\xi) \cdot \xi) \xi$ the normal field of $f$, while $\xi \rightarrow f_{\text {tan }}(\xi)=f-f_{\text {nor }}(\xi)$, is called the tangential field of $f$. Obviously, $f(\xi)=f_{\text {nor }}(\xi)+f_{\text {tan }}(\xi)$ and $f_{\text {nor }}(\xi) \cdot f_{\tan }(\xi)=0$. Furthermore, for $f, g \in \mathrm{c}^{(0)}(\Omega)$ and $\xi \in \Omega, f(\xi) \cdot g(\xi)=f_{\text {nor }}(\xi) \cdot g_{\text {nor }}(\xi)+f_{\tan }(\xi) \cdot g_{\tan }(\xi)$.

Lemma 4.1 The tangential field of $f$ vanishes, i.e., $f_{\tan }(\xi)=0, \xi \in \Omega$, if and only if $f(\xi) \cdot \hat{\tau}(\xi)=0$ for every unit vector $\hat{\tau}(\xi)$ that is perpendicular to $\xi$, i.e., for which $\xi \cdot \hat{\tau}(\xi)=0, \xi \in \Omega$.

Proof. First, assume $f_{\tan }=0$. Then for all $\xi \in \Omega$, we have $f(\xi) \cdot \hat{\tau}(\xi)=0$. Conversely, assume that the tangential field is non-vanishing, i.e., $f_{\tan }(\xi)=f(\xi)-(f(\xi) \cdot \xi) \xi \neq 0$. Then it follows that $f_{\tan }(\xi)\left|f_{\tan }(\xi)\right|^{-1}$ is a unit vector field perpendicular to $\xi$. Hence, by our hypothesis, $f_{\tan }(\xi) \cdot f_{\tan }(\xi)\left|f_{\tan }(\xi)\right|^{-1}=0$. This implies $\left|f_{\tan }(\xi)\right|=0$ which is a contradiction. Thus it follows that $f_{\tan }(\xi)=0$, as required.

Lemma 4.2 Suppose that $f$ is continuous on a simply connected normal region $\Gamma \subset \Omega$. Moreover, let

$$
\int_{\mathcal{C}} \tau_{\xi} \cdot f(\xi) d \sigma(\xi)=0
$$

for every curve $\mathcal{C}$ on $\Gamma$. Then $f_{\tan }(\xi)=0$ for all $\xi \in \Gamma$, i.e., the tangential field of $f$ vanishes for all $\xi \in \Gamma$.

Proof. Choose any point $\xi_{0} \in \Gamma$. Let $\tau_{\xi_{0}}$ be any unit vector satisfying $\tau_{\xi_{0}} \cdot \xi_{0}=0$. Then there is a curve $\mathcal{C}$ on $\Gamma$ passing through $\xi_{0}$ whose unit tangent vector at $\xi_{0}$ is just $\tau_{\xi_{0}}$. Let $\mathcal{C}_{\text {sub }}^{\xi_{0}}$ be any subset of $\mathcal{C}$ containing $\xi_{0}$. Then, in accordance with our assumption,

$$
\int_{\mathcal{C}_{\mathrm{sub}}^{\xi_{0}}} \tau_{\xi} \cdot f(\xi) d \sigma(\xi)=0 .
$$

Hence, letting the length of $\mathcal{C}_{\text {sub }}^{\xi_{0}}$ tend to zero we find $\tau_{\xi_{0}} \cdot f\left(\xi_{0}\right)=0$. Lemma 4.1 then yields $f_{\tan }\left(\xi_{0}\right)=f\left(\xi_{0}\right)-\left(f\left(\xi_{0}\right) \cdot \xi_{0}\right) \xi_{0}=0$. Since $\xi_{0}$ can be any point on $\Gamma$, we have $f_{\tan }(\xi)=f(\xi)-(f(\xi) \cdot \xi) \xi=0$ for all $\xi \in \Gamma$. This is the desired result.

The surface gradient acts like an ordinary gradient in $\mathbb{R}^{3}$ when we integrate it along lines on $\Gamma$. In more detail, suppose $F$ is continuously differentiable in an open set in $\mathbb{R}^{3}$ containing $\Gamma$, and $\mathcal{C}$ is any curve lying on $\Gamma$, starting at $\xi_{0}$ and ending at $\xi_{1}$. Suppose that $\tau_{\xi}$ is the unit tangent vector at $\xi$ on $\mathcal{C}$ pointing from $\xi_{0}$ to $\xi_{1}$. Then

$$
F\left(\xi_{1}\right)-F\left(\xi_{0}\right)=\int_{\mathcal{C}} \tau_{\xi} \cdot \nabla_{\xi}^{*} F(\xi) d \sigma(\xi)
$$

(observe that $\tau_{\xi} \cdot \nabla_{\xi} F(\xi)=\tau_{\xi} \cdot \nabla_{\xi}^{*} F(\xi), \xi \in \Gamma$, see, e.g., [2, 4]). This result enables us to show the following lemma.

Lemma 4.3 Suppose that $\Gamma$ is a simply connected normal region. Let $F$ be of class $\mathrm{C}^{(1)}(\Gamma)$, then $\nabla_{\xi}^{*} F(\xi)=0$ if and only if $F$ is constant.

Proof. If $\nabla_{\xi}^{*} F(\xi)=0$, then we obtain, in connection with (4.1), $F\left(\xi_{1}\right)=F\left(\xi_{0}\right)$ for any $\xi_{0}, \xi_{1}$ on $\Gamma$. Conversely, if $F$ is constant, the identity (4.1) shows that $f=\nabla^{*} F$ fulfills

$$
\int_{\mathcal{C}} \tau_{\xi} \cdot f(\xi) d \sigma(\xi)=0
$$

for every curve $\mathcal{C}$ lying on $\Gamma$. Consequently, following Lemma 4.2, $f_{\tan }(\xi)=0$ for all $\xi \in \Gamma$. This shows that $f_{\tan }(\xi)=$ $f(\xi)-(f(\xi) \cdot \xi) \xi=f(\xi)=\nabla_{\xi}^{*} F(\xi)=0$ for all $\xi \in \Gamma$.

Next we prove the following result of spherical vector analysis (see, e.g., [2]).

Lemma 4.4 Let $f \in \mathrm{c}^{(0)}(\Gamma)$ be a tangent vector field on a simply connected region $\Gamma$, i.e., $f(\xi)=f_{\tan }(\xi), \xi \in \Gamma$. Furthermore, suppose that

$$
\int_{\mathcal{C}} \tau_{\xi} \cdot f(\xi) d \sigma(\xi)=0
$$

for every closed curve on $\Gamma$. Then there is a scalar field $P$ on $\Gamma$ such that

$$
f(\xi)=\nabla_{\xi}^{*} P(\xi), \quad \xi \in \Gamma .
$$

The field $P$ is continuously differentiable and is unique up to a constant. 
Proof. Take an arbitrary, but fixed $\xi_{0} \in \Gamma$. We let

$$
P(\xi)=\int_{\xi_{0}}^{\xi} \tau_{\zeta} \cdot f(\zeta) d \sigma(\zeta)
$$

be the integral along any curve $\mathcal{C}$ that starts at $\xi_{0} \in \Gamma$ and ends at $\xi \in \Gamma$. Then, for any two points $\xi_{0}, \xi$ on $\Gamma$ and any curve $\mathcal{C}$ lying on $\Gamma$ and starting at $\xi_{0}$ and ending at $\xi_{1}$,

$$
P\left(\xi_{1}\right)-P\left(\xi_{0}\right)=\int_{\xi_{0}}^{\xi_{1}} \tau_{\zeta} \cdot f(\zeta) d \sigma(\zeta) .
$$

Observing (4.1) we find

$$
P\left(\xi_{1}\right)-P\left(\xi_{0}\right)=\int_{\xi_{0}}^{\xi_{1}} \tau_{\zeta} \cdot \nabla_{\zeta}^{*} P(\zeta) d \sigma(\zeta) .
$$

Combining (4.2) and (4.3) we obtain

$$
\int_{\xi_{0}}^{\xi_{1}} \tau_{\zeta} \cdot\left(f(\zeta)-\nabla_{\zeta}^{*} P(\zeta)\right) d \sigma(\zeta)=0
$$

for any curve $\mathcal{C}$ on $\Gamma$. Lemma 4.2, therefore, shows us that $f(\xi)-\nabla_{\xi}^{*} P(\xi)=0, \xi \in \Gamma$. The proof that $P$ is continuously differentiable on $\Gamma$ is omitted. The easiest way to construct such a proof is to take $P$ constant on each straight line passing through $\Gamma$ in the normal direction (see, e.g., [2]). In order to verify that $P$ is unique up to a constant, we observe that $\nabla_{\xi}^{*} P_{1}(\xi)=\nabla_{\xi}^{*} P_{2}(\xi), \xi \in \Gamma$, implies $\nabla_{\xi}^{*}\left(P_{1}-P_{2}\right)(\xi)=0, \xi \in \Gamma$, i.e., by virtue of Lemma $4.3, P_{1}-P_{2}=$ const.

Now we are able to verify the following important theorem.

Theorem 4.5 Let $f \in \mathrm{c}^{(1)}(\Gamma)$ be a tangential field on a simply connected normal region $\Gamma$, i.e., $f(\xi)=f_{\tan }(\xi)$ for all $\xi \in \Gamma$. Then $L_{\xi}^{*} \cdot f(\xi)=0, \xi \in \Gamma$, if and only if there is a scalar field $P$ such that

$$
f(\xi)=\nabla_{\xi}^{*} P(\xi), \quad \xi \in \Gamma,
$$

and $P$ is unique up to an additive constant ( $P$ is called potential function for $f$ ).

Similarly, $\nabla_{\xi}^{*} \cdot f(\xi)=0, \xi \in \Gamma$, if and only if there is a scalar field $S$ such that

$$
f(\xi)=L_{\xi}^{*} S(\xi), \quad \xi \in \Gamma,
$$

and $S$ is unique up to an additive constant ( $S$ is called stream function for $f$ ).

Proof. The condition $f=\nabla^{*} P$ implies $L^{*} \cdot f=0$, and $f=L^{*} S$ implies $\nabla^{*} \cdot f=0$.

Conversely, assume that $L_{\xi}^{*} \cdot f(\xi)=0, \xi \in \Gamma$. Then the surface theorem of Stokes implies

$$
\int_{\mathcal{C}} \tau_{\xi} \cdot f(\xi) d \sigma(\xi)=0
$$

for every closed curve $\mathcal{C}$ on $\Gamma$. From Lemma 4.4 it follows that there exists a scalar field $P$ such that $f=\nabla^{*} P$. Furthermore, $P$ is unique up to an additive constant.

Finally, suppose $\nabla^{*} \cdot f=0$. Then $L_{\xi}^{*} \cdot(\xi \wedge f(\xi))=0, \xi \in \Gamma$, hence, there is a scalar field $S$, unique up to a constant, such that $-\xi \wedge f(\xi)=\nabla_{\xi}^{*} S(\xi), \xi \in \Gamma$. This is equivalent to $-\xi \wedge(\xi \wedge f(\xi))=\left(\xi \wedge \nabla_{\xi}^{*}\right) S(\xi), \quad \xi \in \Gamma$, or $f=L^{*} S$ on $\Gamma$. This proves Theorem 4.5 .

From Lemma 4.3 we are immediately able to deduce the following statement.

Lemma 4.6 Let $F$ be of class $C^{(1)}(\Gamma)$, then $L_{\xi}^{*} F(\xi)=0$ if and only if $F$ is constant.

Proof. If $L_{\xi}^{*} F(\xi)=0$, i.e., $\xi \wedge \nabla_{\xi}^{*} F(\xi)=0$ for all $\xi \in \Gamma$. Then $\xi \wedge \xi \wedge \nabla_{\xi}^{*} F(\xi)=\xi \cdot \nabla_{\xi}^{*} F(\xi)-\nabla_{\xi}^{*} F(\xi)(\xi \cdot \xi)=$ $-\nabla_{\xi}^{*} F(\xi)=0$ for all $\xi \in \Gamma$. Thus by virtue of Lemma 4.3 we find $F=$ const.

Conversely, if $F$ is constant, then $L_{\xi}^{*} F(\xi)=\xi \wedge \nabla_{\xi}^{*} F(\xi)=0$ for all $\xi \in \Gamma$. This proves our assertion. 


\section{The Differential Equations of the Surface Curl Gradient}

In what follows we give two different solvability conditions for the differential equation (1.1) of the surface curl gradient $L^{*}$. First, based on the results of Chapter 3 we formulate a certain integrability condition to assure uniqueness.

Theorem 5.1 Given $f=L^{*} F \in c^{(0)}(\Omega)$. Then the scalar function $F$ is uniquely determined by the condition:

$$
\frac{1}{4 \pi} \int_{\Omega} F(\eta) d \omega(\eta)=C_{0}, \quad C_{0} \in \mathbb{R}
$$

Proof. We suppose that $F_{1}, F_{2} \in C^{(1)}(\Omega)$ are functions satisfying the condition above. Then the difference $D=$ $F_{1}-F_{2}$ satisfies $L^{*} D=0$ on $\Omega$ and $\frac{1}{4 \pi} \int_{\Omega} D(\eta) d \omega(\eta)=0$. Consequently, from Corollary 3.4, we obtain $D(\xi)=0$ for all $\xi \in \Omega$. Therefore, $F_{1}=F_{2}$, as required.

Theorem 5.2 Let $\Gamma \subset \Omega$ be a simply connected normal region. Given $f=L^{*} F \in c^{(0)}(\Omega)$. Then the scalar function $F$ is uniquely determined by the condition taken at one point $\xi_{0} \in \bar{\Gamma}$ :

$$
\frac{1}{4 \pi} \int_{\Gamma} F(\eta) d \omega(\eta)+\int_{\partial \Gamma} F(\eta) \tau_{\eta} \cdot G\left(\Delta^{*} ; \xi_{0}, \eta\right) d \sigma(\eta)=C_{0}, \quad C_{0} \in \mathbb{R} .
$$

Proof. We look at the difference $D$ of two solutions which satisfies $L^{*} D=0$ in $\Gamma$. By Lemma 4.6 we find $D(\xi)=$ const $=\mathrm{C}$ for all $\xi \in \bar{\Gamma}$. In connection with Theorem 3.3 we have

$$
\frac{C}{2 \pi}\left(\frac{\|\Gamma\|}{2}+2 \pi \int_{\partial \Gamma} \tau_{\eta} \cdot L_{\eta}^{*} G\left(\Delta^{*} ; \xi_{0}, \eta\right) d \sigma(\eta)\right)=0
$$

Using with (3.6) we, therefore, find $C=0$, i.e., $D=0$ on $\bar{\Gamma}$, as required.

Second, based on the results of Chapter 4, we are able to formulate a uniqueness condition by fixing a certain functional value.

Theorem 5.3 Given $f=L^{*} F \in c^{(0)}(\Omega)$. Then the scalar function $F$ is uniquely determined by the condition taken at one point $\xi_{0} \in \Omega$ :

$$
F\left(\xi_{0}\right)=C_{0}, \quad C_{0} \in \mathbb{R} .
$$

Proof. The constant difference $D$ of two functions satisfying the conditions is equal to $D\left(\xi_{0}\right)=C_{0}-C_{0}=0=D(\xi)$ for all $\xi \in \Omega$.

Theorem 5.4 Suppose that $\Gamma$ is a simply connected normal region. Given $f=L^{*} F \in c^{(0)}(\Omega)$. Then the scalar function $F$ is uniquely determined by the condition taken at one point $\xi_{0} \in \bar{\Gamma}$ :

$$
F\left(\xi_{0}\right)=C_{0}, \quad C_{0} \in \mathbb{R} .
$$

Proof. $D$ is constant on $\bar{\Gamma}$ with $D\left(\xi_{0}\right)=0$. Hence $D\left(\xi_{0}\right)=0$ in $\bar{\Gamma}$.

\section{Regularized Green's Theorems on (Normal) Regions on the Sphere}

In the following we first introduce the regularized Green function with respect to $\Delta^{*}$. We state its definition together with some properties to be needed for the discussion of spherical wavelets on regular regions.

Definition 6.1 Given $\rho \in(0,2)$, the regularized Green function with respect to $\Delta^{*}$ is defined for all $\xi, \eta \in \Omega$ by

$$
G_{\rho}\left(\Delta^{*} ; \xi, \eta\right)= \begin{cases}\frac{1}{4 \pi} \ln (1-\xi \cdot \eta)+\frac{1}{4 \pi}-\frac{1}{4 \pi} \ln 2, & 1-\xi \cdot \eta>\rho, \\ \frac{1}{4 \pi \rho}(1-\xi \cdot \eta)+\frac{1}{4 \pi} \ln (\rho)-\frac{1}{4 \pi} \ln 2, & 1-\xi \cdot \eta \leq \rho .\end{cases}
$$

The regularized Green function with respect to the Beltrami operator $(\xi, \eta) \rightarrow G_{\rho}\left(\Delta^{*} ; \xi, \eta\right)$ only depends on the inner product of $\xi$ and $\eta$, hence, it is a radial basis function, i.e., $G_{\rho}\left(\Delta^{*} ; \mathbf{t} \xi, \mathbf{t} \eta\right)=G_{\rho}\left(\Delta^{*} ; \xi, \eta\right)$ holds true for all orthogonal transformations $\mathbf{t}$. Figure 1 gives an illustration of the regularized Green function with respect to $\Delta^{*}$. Note that, by construction, this kernel function represents an approximation of the original Green's function, i.e., it converges pointwise to Green's function as $\rho$ tends to 0 . 


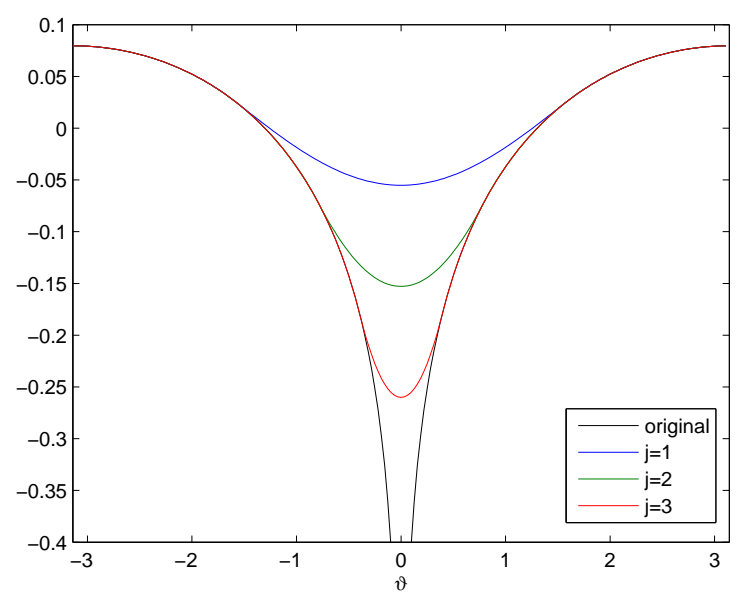

Fig. 1 The regularized Green function $\vartheta \mapsto G_{\rho}\left(\Delta^{*} ; \cos \vartheta\right)$ for $\rho=1-\cos \left(\pi 2^{-j}\right)$ with $j=1,2,3$ and the original Green function $G_{\rho}\left(\Delta^{*} ; \xi \cdot \eta\right)$ with respect to the Beltrami operator $\Delta^{*}$. Note that $\xi \cdot \eta=\cos \vartheta, \vartheta=\measuredangle(\xi, \eta)$.

We immediately realize that the regularized Green function with respect to $\Delta^{*}$ is continuously differentiable. Applying the surface curl gradient $L^{*}$ to the second variable yields to the so-called regularized Green function with respect to $L^{*}$. Obviously, for $\rho \in(0,2)$, we obtain for all $\xi, \eta \in \Omega$

$$
g_{\rho}^{L^{*}}(\xi, \eta)=L_{\eta}^{*} G_{\rho}\left(\Delta^{*} ; \xi, \eta\right)= \begin{cases}\frac{1}{4 \pi} \frac{1}{1-\xi \cdot \eta}(\xi \wedge \eta), & 1-\xi \cdot \eta>\rho, \\ \frac{1}{4 \pi} \frac{1}{\rho}(\xi \wedge \eta), & 1-\xi \cdot \eta \leq \rho .\end{cases}
$$

Observing the equation $|\xi \wedge \eta|=\sqrt{1-(\xi \cdot \eta)^{2}}$ we derive for all $\xi, \eta \in \Omega$ and $\rho \in(0,2)$

$$
\left|g_{\rho}^{L^{*}}(\xi, \eta)\right|= \begin{cases}\frac{1}{4 \pi} \sqrt{\frac{1+\xi \cdot \eta}{1-\xi \cdot \eta}}, & 1-\xi \cdot \eta>\rho \\ \frac{1}{4 \pi} \sqrt{1-(\xi \cdot \eta)^{2}}, & 1-\xi \cdot \eta \leq \rho\end{cases}
$$

A graphical impression of the norm of the regularized Green function with respect to $L^{*}$ and the norm of the surface curl gradient of Green's function with respect to $\Delta^{*}$ is illustrated in Figure 2. By similar arguments as known from potential theory (see e.g. $[6,9]$ ) we obtain the following counterpart of the integral formula developed in Chapter 3.

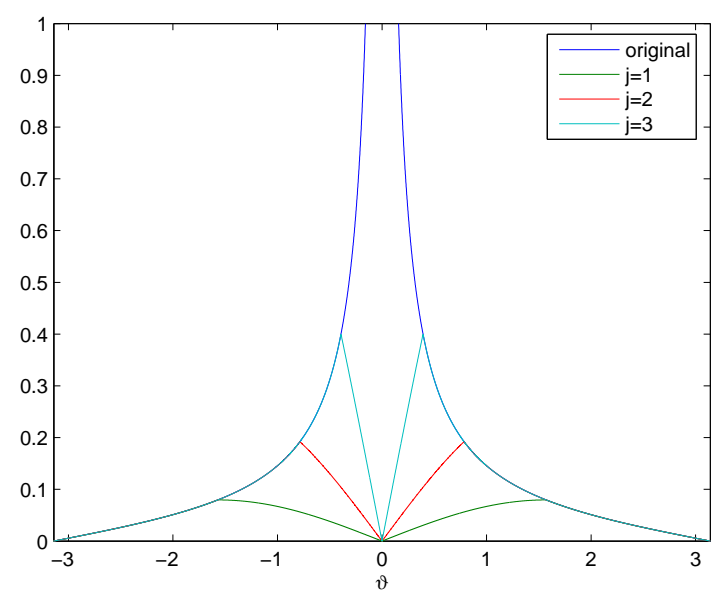

Fig. 2 The norm of the regularized Green function $\vartheta \rightarrow\left|g_{\rho}^{L^{*}}(\cos (\vartheta))\right|$ for $\rho=1-\cos \left(\pi 2^{-j}\right)$ with $j=1,2,3$ and the norm of the surface curl gradient of Green's function with respect to $\Delta^{*}$.

Theorem 6.2 For $F \in C^{(1)}(\Omega)$ we have

$$
\lim _{\rho \rightarrow 0} \sup _{\xi \in \Omega}\left|F(\xi)-\frac{1}{4 \pi} \int_{\Omega} F(\eta) d \omega(\eta)-\int_{\Omega} g_{\rho}^{L^{*}}(\xi, \eta) \cdot L^{*} F(\eta) d \omega(\eta)\right|=0 .
$$


After deriving the regularized version of the integral theorem for $L^{*}$ on $\Omega$ we now turn to the regularized integral theorem for $L^{*}$ on normal regions $\Gamma$. For that purpose we introduce the following settings.

Definition 6.3 Let $\Gamma \subset \Omega$ be a normal region of the unit sphere $\Omega$. For $F \in C^{(1)}(\bar{\Gamma})$ we let

$$
S_{\rho}(F)(\xi)=\int_{\Gamma} g_{\rho}^{L^{*}}(\xi, \eta) \cdot L_{\eta}^{*} F(\eta) d \omega(\eta)-\int_{\partial \Gamma} g_{\rho}^{L^{*}}(\xi, \eta) \cdot F(\eta) \tau_{\eta} d \sigma(\eta), \quad \rho \in(0,2),
$$

as a counterpart of

$$
S(F)(\xi)=\int_{\Gamma} L_{\eta}^{*} G\left(\Delta^{*} ; \xi, \eta\right) \cdot L_{\eta}^{*} F(\eta) d \omega(\eta)-\int_{\partial \Gamma} L_{\eta}^{*} G\left(\Delta^{*} ; \xi, \eta\right) \cdot F(\eta) \tau_{\eta} d \sigma(\eta) .
$$

Clearly, it is not hard to show that

$$
\lim _{\rho \rightarrow 0} \sup _{\xi \in \Omega}\left|S(F)(\xi)-S_{\rho}(F)(\xi)\right|=0 .
$$

Theorem 6.4 (Regularized Integral Theorem for $L^{*}$ on $\Gamma$ ) Let $\Gamma \subset \Omega$ be a normal region with boundary $\partial \Gamma$. Suppose that $F$ is a continuously differentiable function on $\bar{\Gamma}$, i.e., $F \in C^{(1)}(\bar{\Gamma})$. Then

$$
\lim _{\rho \rightarrow 0} \sup _{\xi \in \bar{\Gamma}}\left|\frac{\alpha(\xi)}{2 \pi} F(\xi)-\frac{1}{4 \pi} \int_{\Gamma} F(\eta) d \omega(\eta)+S_{\rho}(F)(\xi)\right|=0
$$

where $\alpha(\xi)$ denotes, as usually, the solid angle subtended at $\xi \in \bar{\Gamma}$.

\section{Vector Spherical Wavelets on Normal Regions}

We turn our attention to the introduction of vector spherical wavelets, where $\Gamma \subset \Omega$ is supposed to be a normal region. We choose a sequence which divides the continuous scale interval $(0,2)$ into discrete pieces. More explicitly, $\left(\rho_{j}\right)_{j \in \mathbb{N}_{0}}$ denotes a sequence of real numbers satisfying $\lim _{j \rightarrow \infty} \rho_{j}=0$ and $\lim _{j \rightarrow 0} \rho_{j}=1$. For example, we can choose $\rho_{j}=2^{-j}$ or $\rho_{j}=1-\cos \left(\pi 2^{-j}\right), j \in \mathbb{N}_{0}$. The point of departure for our considerations on normal regions $\Gamma$ is Theorem 6.4 in the form

$$
\frac{\alpha(\xi)}{2 \pi} F(\xi)-F_{\text {mean }}^{\Gamma}=-\lim _{j \rightarrow \infty} S_{\rho_{j}}(F)(\xi), \text { where } F_{\text {mean }}^{\Gamma}=\frac{1}{4 \pi} \int_{\Gamma} F(\eta) d \omega(\eta), \quad \xi \in \bar{\Gamma} .
$$

Note that the discrete steps in this approximation process are called scales, i.e., the value $j$ takes the role of the scale parameter, i.e., the "zooming-in" parameter. By using discrete regularization parameters we are naturally led to the following type of scale discretized Green wavelets.

Definition 7.1 Let $\left\{g_{\rho_{j}}^{L^{*}}\right\}_{j \in \mathbb{N}_{0}}$ be the regularized Green function with respect to $L^{*}$ (see (6.1)). Then the scale discretized regularized Green wavelet function with respect to $L^{*}$ is defined by

$$
\begin{aligned}
\psi_{\rho_{j}} & =g_{\rho_{j+1}}^{L^{*}}-g_{\rho_{j}}^{L^{*}} \\
& =L_{\eta}^{*} G_{\rho_{j+1}}\left(\Delta^{*} ; \xi, \eta\right)-L_{\eta}^{*} G_{\rho_{j}}\left(\Delta^{*} ; \xi, \eta\right), \quad j \in \mathbb{N}_{0} .
\end{aligned}
$$

In fact, the difference of two consecutive scales of regularized Green functions with respect to $\Delta^{*}$ reads

$$
\begin{aligned}
& G_{\rho_{j+1}}\left(\Delta^{*} ; \xi, \eta\right)-G_{\rho_{j}}\left(\Delta^{*} ; \xi, \eta\right) \\
& =\left\{\begin{array}{cr}
0, & 1-\xi \cdot \eta \quad>\rho_{j}, \\
\frac{1}{4 \pi} \ln (1-\xi \cdot \eta)-\frac{1}{4 \pi \rho_{j}}(1-1-\xi \cdot \eta)+\frac{1}{4 \pi}\left(1-\ln \left(\rho_{j}\right)\right), \quad \rho_{j}>1-\xi \cdot \eta>\rho_{j+1}, & 1- \\
\left(\frac{1}{4 \pi \rho_{j+1}}-\frac{1}{4 \pi \rho_{j}}\right)(1-\xi \cdot \eta)+\frac{1}{4 \pi}\left(\ln \left(\rho_{j+1}\right)-\ln \left(\rho_{j}\right)\right), & 1-\xi \cdot \eta \leq \rho_{j+1},
\end{array}\right.
\end{aligned}
$$

such that

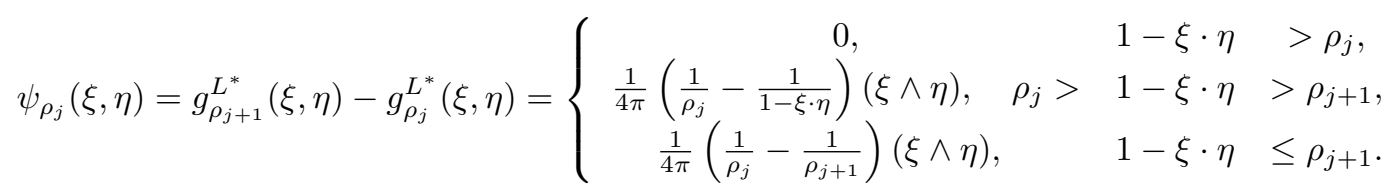

A graph of the norm of the scale discretized regularized Green wavelet function with respect to $L^{*}$ for the discretization parameters $\rho=1-\cos \left(\pi 2^{-j}\right)$ with $j=0,1,2,3$ is shown in Figure 3. Note, that the functions $\psi_{\rho_{j}}$ have a local support. 


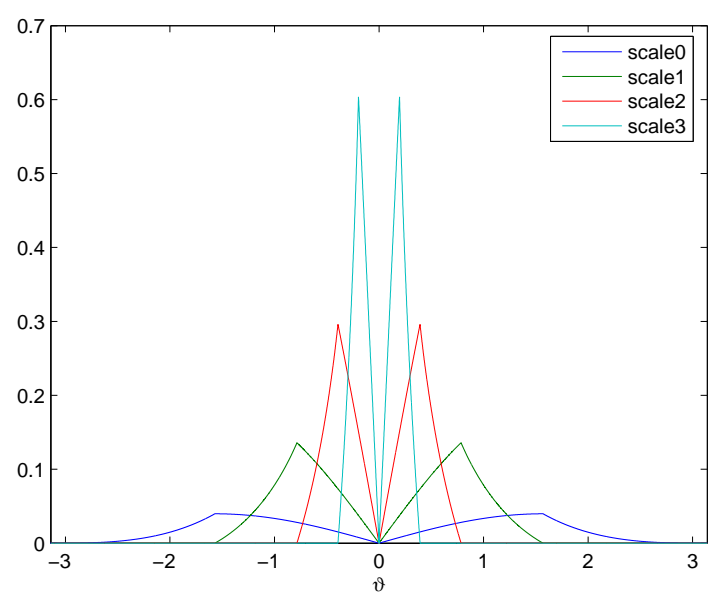

Fig. 3 The norm of the regularized Green wavelet function $\vartheta \rightarrow\left|\Psi_{\rho_{j}}(\cos (\vartheta))\right|$ with respect to $L^{*}$ for $\rho=1-\cos \left(\pi 2^{-j}\right)$ with scale $j=0,1,2,3$.

$S_{\rho_{j}}(F)(\xi)$, as given by Definition 6.3, is called the scale discrete regularized Green scaling function transform with respect to $L^{*}$. Let $\left\{\psi_{\rho_{j}}\right\}_{\mathrm{J} \in \mathbb{N}_{0}}$ be the scale discretized regularized Green function with respect to $L^{*}$. The scale discretized regularized Green wavelet transform with respect to $L^{*}$ is defined by

$$
W_{\rho_{j}}(F)(\xi)=\int_{\Gamma} L_{\eta}^{*} F(\eta) \cdot \psi_{\rho_{j}}(\xi, \eta) d \omega(\eta)-\int_{\partial \Gamma} F(\eta) \tau_{\eta} \cdot \psi_{\rho_{j}}(\xi, \eta) d \sigma(\eta) .
$$

We arrive at the following theorem.

Theorem 7.2 Let $\left\{g_{\rho_{j}}^{L^{*}}\right\}_{j \in \mathbb{N}_{0}}$ be the regularized Green function with respect to $L^{*}$. Then the multiscale reconstruction of a function $F \in C^{(1)}(\Gamma)$ is given by

$$
\frac{\alpha(\xi)}{2 \pi} F(\xi)-F_{\text {mean }}^{\Gamma}=\sum_{j=-\infty}^{\infty} W_{\rho_{j}}(F)(\xi), \quad \xi \in \Gamma
$$

where the equality holds in the $\|\cdot\|_{C(\bar{\Gamma})}$-sense.

By observing the definition of the scaling transform $S_{\rho_{j}}(F)(\xi)$, Theorem 7.2 admits the following reformulation.

Corollary 7.3 Under the assumptions of Theorem 7.2

$$
\frac{\alpha(\xi)}{2 \pi} F(\xi)-F_{\text {mean }}^{\Gamma}-S_{\rho_{J}}(F)(\xi)=\sum_{j=J}^{\infty} W_{\rho_{j}}(F)(\xi), \quad \xi \in \bar{\Gamma},
$$

for every $J \in \mathbb{N}_{0}$ in the $\|\cdot\|_{C(\bar{\Gamma})}$-sense.

These reconstruction formula will now be applied to the modelling of oceanic circulation.

\section{Modelling of Sea Surface Topography From Ocean Circulation}

As is well known, the (spherical) Earth $\Omega$ can de decomposed uniquely into two normal regions, namely the oceanic part $\Omega^{o c}$ and the continental part $\Omega^{c o}$. Due to the definition of the sea surface topography we assume the differential equation $L^{*} H=h$ to be valid on $\Omega^{o c}$ thereby relating the sea surface topography $H \in C^{(1)}\left(\overline{\Omega^{o c}}\right)$ to the geostrophic ocean flow $h \in C^{(0)}(\bar{\Gamma})$. Moreover, we assume that $H=0$ on $\partial \Omega^{o c}$ and $\int_{\Omega^{o c}} H(\xi) d \omega(\xi)=0$. Summarizing our results for the normal region $\Gamma=\Omega^{o c}$, we therefore obtain

$$
H(\xi)=-\int_{\Omega^{o c}} L_{\eta}^{*} G\left(\Delta^{*} ; \xi, \eta\right) \cdot h(\eta) d \omega(\eta), \quad \xi \in \Omega^{o c}
$$

which can be approximated as follows

$$
H(\xi)=-\lim _{j \rightarrow \infty} \int_{\Omega^{o c}} L_{\eta}^{*} G_{\rho_{j}}\left(\Delta^{*} ; \xi, \eta\right) \cdot h(\eta) d \omega(\eta), \quad \xi \in \Omega^{o c}
$$


It suffices to have an (in the sense of Weyl) equidistributed nodal set $\left(\eta_{i}, h\left(\eta_{i}\right)\right), \eta_{i} \in \overline{\Omega^{o c}}, i=1, \cdots, N$, to discretize the integral on the left hand side and to establish a multiscale approximation of the sea surface topography.

In practice, however, we are often confronted with the problem of determining the sea surface topography on a certain subdomain $\Gamma$ of $\Omega^{o c}$ (e.g., caps, squares or rectangles), where suitable discrete data information about the velocity vectors of ocean currents is available. When we are interested in solving that problem numerically from discrete data material our approach shows that we have to know, in addition, the sea surface topography on the boundary $\partial \Gamma$. But this quantity does not vanish generally. Even more, our numerical calculation based on discrete data is only unique up to a constant $H_{m e a n}^{\Gamma}$ :

$$
\frac{\alpha(\xi)}{2 \pi} H(\xi)-H_{\text {mean }}^{\Gamma}=-\lim _{j \rightarrow \infty}\left(\int_{\Gamma} h(\eta) \cdot g_{\rho_{j}}^{L^{*}}(\xi, \eta) d \omega(\eta)+\int_{\partial \Gamma} H(\eta) \tau_{\eta} \cdot g_{\rho_{j}}^{L^{*}}(\xi, \eta) d \sigma(\eta)\right) .
$$

In what follows, particular attention is paid to the numerical stability caused by the specific observation of the boundary terms in our calculation. Two examples will be presented for the discrete "Topography Problem" involving discrete data on the boundary $\partial \Gamma$. First, we consider a spherical cap $\Gamma$ as a regular region. In a second step we will have a look at a normal region, i.e., a rectangle.

\subsection{Multiscale Approximation on a Spherical Cap}

The spherical cap under consideration is defined by its center $\zeta \in \Omega$ and its radius $r>0$, more precisely we let

$$
\Gamma_{r}(\zeta)=\{\eta \in \Omega:|\zeta-\eta|<r\} .
$$

In this case the tangential unit vector $\tau_{\eta}$ is explicitly given for all $\eta \in \partial \Gamma_{r}^{s e a}(\zeta)$ by (3.5). Substituting the tangential unit vector in the equations above we obtain

$$
\frac{\alpha(\xi)}{2 \pi} H(\xi)-H_{\text {mean }}^{\Gamma_{r}}=-\lim _{j \rightarrow \infty}\left(\int_{\Gamma_{r}(\zeta)} h(\eta) \cdot g_{\rho_{j}}^{L^{*}}(\xi, \eta) d \omega(\eta)+\int_{\partial \Gamma_{r}(\zeta)} H(\eta) \frac{g_{\rho_{j}}^{L^{*}}(\xi, \eta) \cdot(\zeta \wedge \eta)}{\sqrt{1-(\zeta \cdot \eta)^{2}}} d \sigma(\eta)\right)
$$

To be more precise, the region of interest in our first example is a spherical cap $\Gamma_{21}$ where $21^{\circ}$ denotes the apex angle of the cap. Furthermore, we assume that the velocity field measurements are not continuously given, but on an equiangular longitude-latitude grid with a step size of $0.1^{\circ}$. The sea surface topography is prescribed at a finite set of boundary points that are sampled with an angular distance of $0.0072^{\circ}$. Both data sets have been generated from an artificial topography model, the CLS01 model (which the Geomathematics Group, TU Kaiserslautern, received from the French enterprise CLS (Collecte Localisation Satellites)). It has been computed using a 7-year TOPEX/ POSEIDON mean profile, a 5-year ERS1/2 mean profile, a 2-year GEOSAT mean profile and the two 168-day non repeat cycle data of the ERS-1 geodetic phase. The data were processed and homogenized using the most recent corrections to compute the CLS01 mean sea surface (CLS01 MSS). The surface is derived from altimetric data on oceans and from geoidal undulations elsewhere (continuously connected in between, starting from an ocean depth of 10 meters). Since the velocity as well as the sea surface topography have been generated from the CLS01 model we are able to compare the calculated results with the original data later on. Figure 4 illustrates the mean dynamic topography which is used to calculate the input dataset, i.e., the geostrophic flow which is also shown in Figure 4. Since we are especially interested in boundary effects, we always plot the spherical cap together with its surrounding environment.

We deal with the discretization of the scale interval $(0,2)$. Very often the regularization parameter is set to $\rho_{j}=2^{-j}$, $j \in \mathbb{N}_{0}$. In order to increase the speed of convergence we use the definition $\rho_{j}=1-\cos \left(2^{-2 j}\right), j \in \mathbb{N}_{0}$, instead. The effect is that we obtain a good approximation of the sea surface topography already at low scales, which can be seen in Figure 5 presenting the reconstruction of the topography at lower scales. The left column contains the reconstruction based on the regularized Green function with respect to $L^{*}$ and the right column shows the approximation of the topography with the corresponding scale discretized regularized Green wavelet function.

At first sight the approximated sea surface topography is close to the original topography inside the spherical cap, while the topography at the boundary of the spherical cap seems to be disturbed. Taking the absolute value of the difference between the original and the approximated sea surface topography, we can better specify the approximation errors (see Figure 6).

\subsection{Multiscale Approximation on a Spherical Rectangle}

Next, we will have a look at the aforementioned rectangular region. From the dataset of the spherical cap we cut off a rectangular region and generate a corresponding boundary dataset. Figure 7 illustrates the dynamic topography and the geostrophic flow which is used as input dataset. 

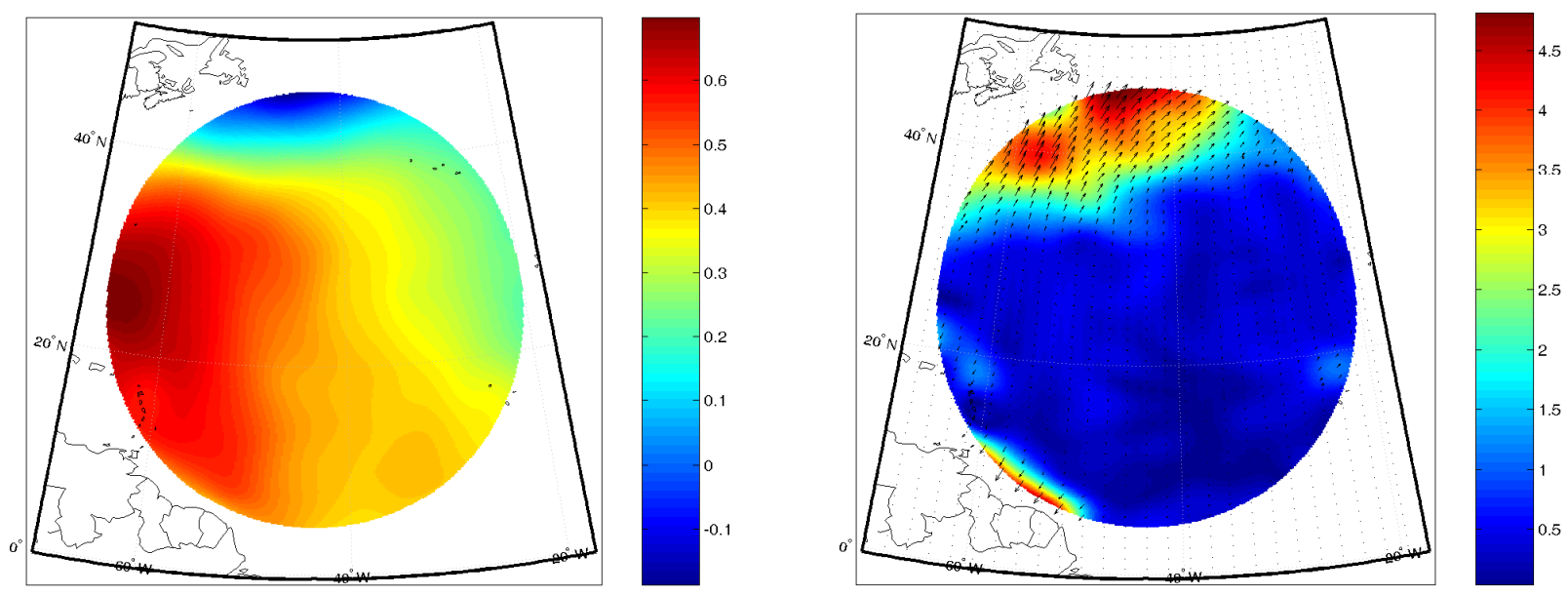

Fig. 4 Plot of the mean dynamic topography in [m] (left), which is used to calculate the input dataset, i.e., the geostrophic flow in [cm/s] (right).

Omitting the vertices in the process of integration it is easy to calculate $\tau_{\eta}$ for each $\eta \in \partial \Gamma$. As in the case of the spherical cap we achieve a good approximation of the sea surface topography, which can be seen in Figure 8 . It shows the reconstruction of the topography at lower scales. The left column contains the reconstruction based on the regularized Green function with respect to $L^{*}$ and the right column shows the approximation of the topography with the corresponding regularized Green wavelet function. Analogously to the case of a spherical cap we achieve small errors.

A question we are interested in is in how far the part of the boundary integral influences the reconstruction. To give an impression of this influence Figure 10 compares the total reconstruction to the part of the reconstruction coming from the boundary integral in the cap and in the rectangular region at scale 5.

It is an interesting fact that the boundary integral takes the major part of the whole reconstruction. The numerical results demonstrate that the new multiscale approximation method based on the regularized Green function with respect to $L^{*}$ yields an efficient procedure to model sea surface topography from ocean velocity field data. Already at low scales, i.e., with larger regularization caps, the approximation error inside of the regular region tends to zero. Compared to standard methods using a truncated velocity field model that neglects the boundary effects our approximation method shows an appealing convergence of the approximated version to the exact solution at the boundary of the region.

\section{References}

[1] D.G. Andrews, An Introduction to Atmospheric Physics. Cambridge University Press, 2000.

[2] G.E. Backus, R. Parker, and C. Constable, Foundations of Geomagnetism. Cambridge University Press, Cambridge, 1996.

[3] W. Freeden, On Integral Formulas of the (Unit) Sphere and Their Application to Numerical Computation of Integrals. Computing, 1980.

[4] W. Freeden, T. Gervens, and M. Schreiner, Constructive Approximation on the Sphere (With Applications to Geomathematics). Oxford Science Publications, Clarendon, 1998.

[5] W. Freeden, D. Michel, and V. Michel. Local Multiscale Approximations of Geostrophic Oceanic Flow: Theoretical Background and Aspects of Scientific Computing. Marine Geodesy, 28, 313-329, 2005.

[6] S. Gramsch, Integral Formulae and Wavelets on Regular Regions of the Sphere. $\mathrm{PhD}$ thesis, Geomathematics Group, University of Kaiserslautern (2006).

[7] E. Groten, Geodesy and the Earth's Gravity Field I,II. Dümmler, 1979.

[8] W.A. Heiskanen and H. Moritz, Physical Geodesy. W.H. Freeman and Company, San Francisco, 1967.

[9] O.D. Kellogg, Foundation of Potential Theory. Springer, Berlin, Heidelberg, New York, 1967.

[10] R.S. Nerem, B.D. Tapley and C.K. Shum. A General Ocean Circulation Model Determined in a Simultaneous Solution with the Earth's Gravity Field. IAG Symposia, 104,158-166, 1989, Eds. Sünkel and Baker.

[11] J. Pedlosky, Geophysical Fluid Dynamics. Springer, 1979.

[12] R.H. Stewart, Introduction to Physical Oceanography. Electronic publication, Texas, USA. A and M University College Station, Texas. 


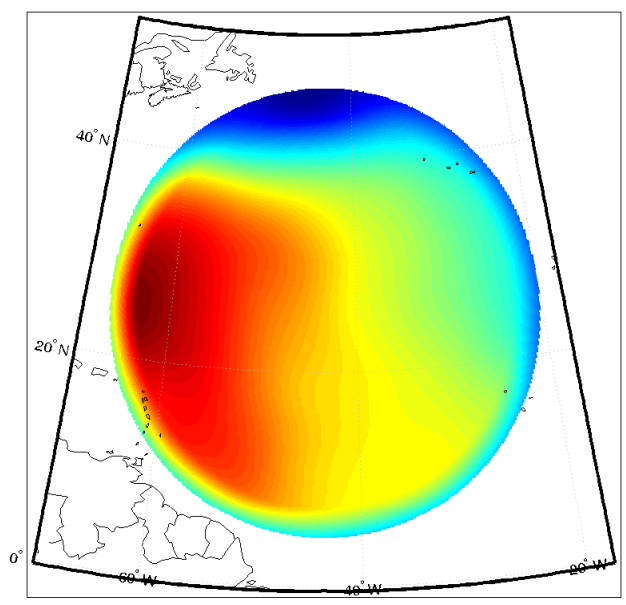

scale 3 (scaling function)

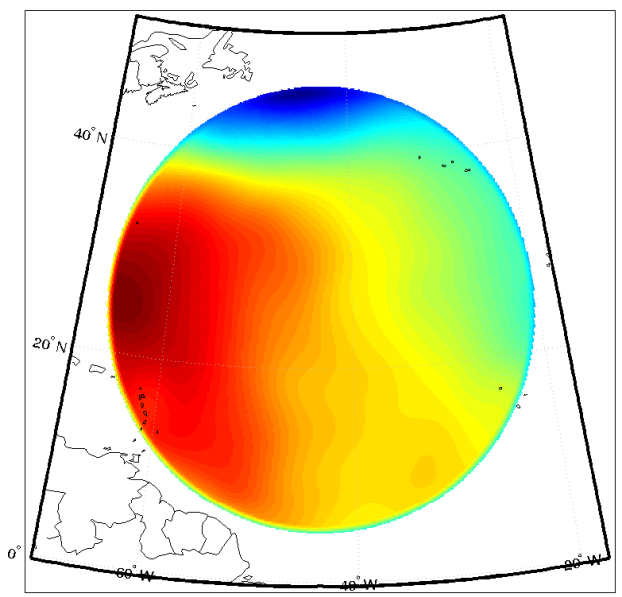

scale 4 (scaling function)

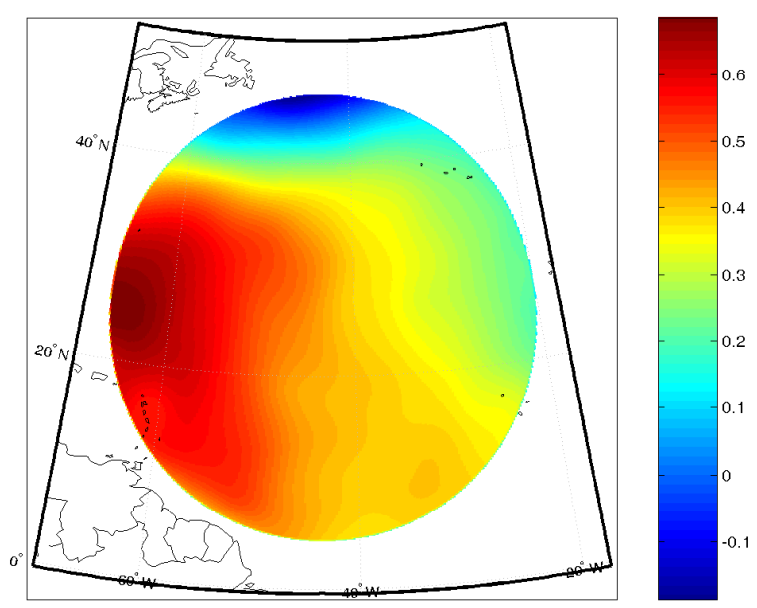

scale 5 (scaling function)

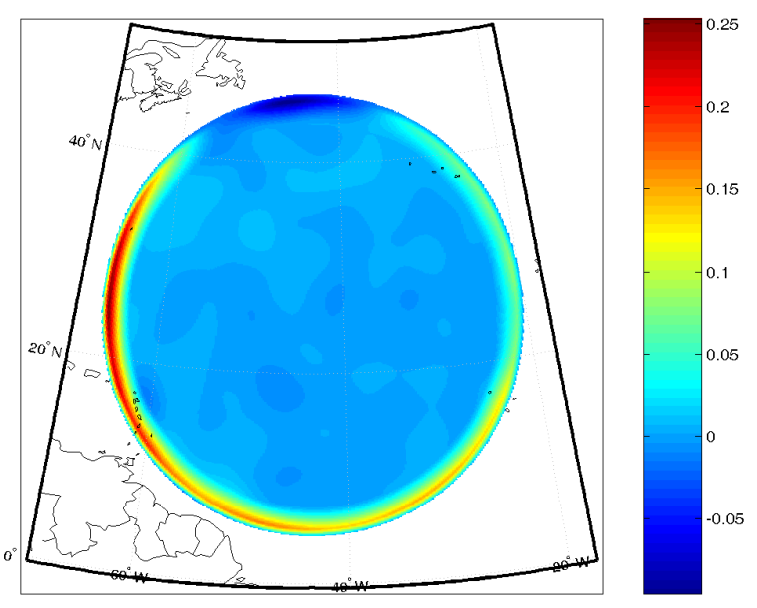

scale 3 (wavelet)

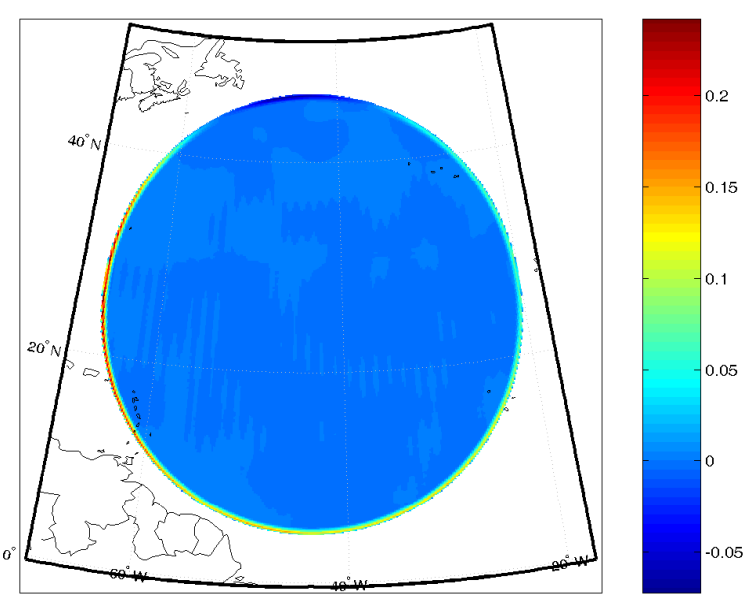

scale 4 (wavelet)
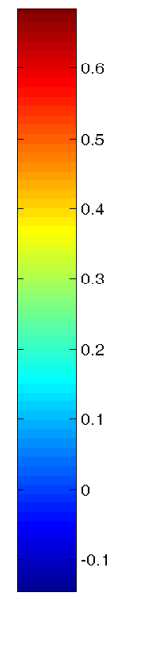


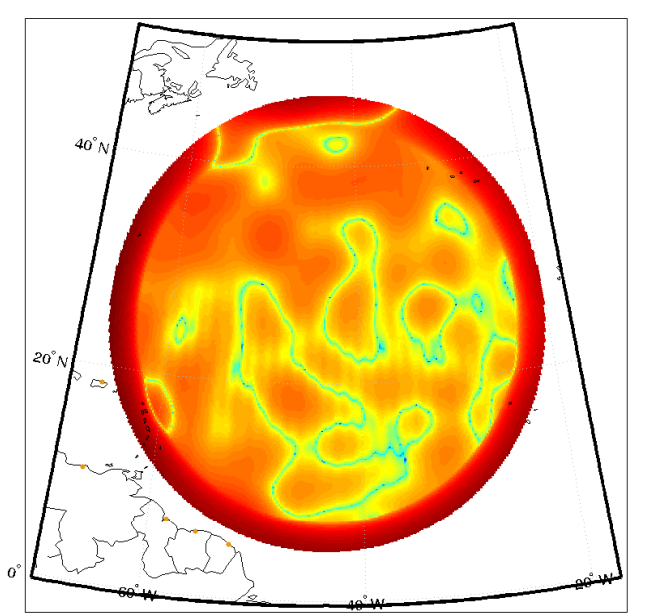

scale 3

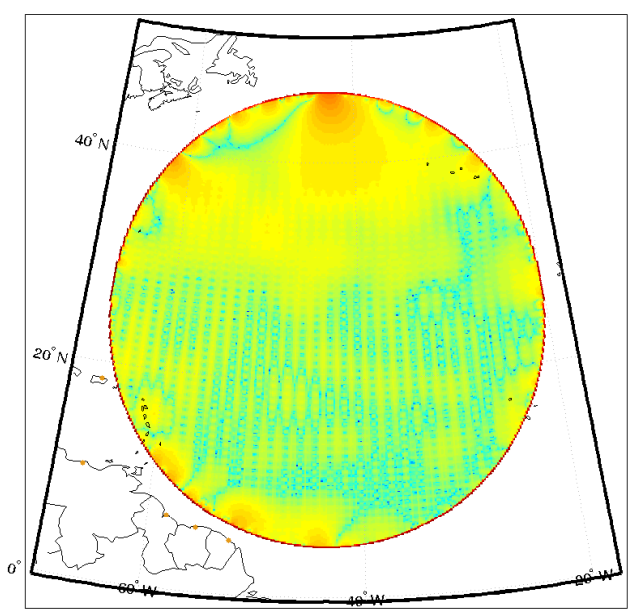

scale 5
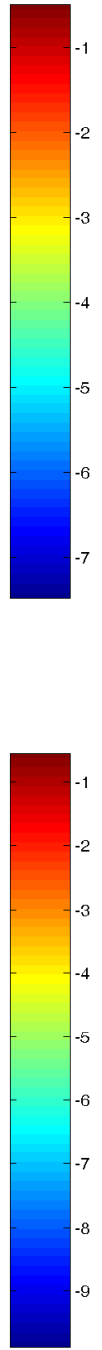

Fig. 6 Approximation error of the dynamic topography in the spherical cap $\Gamma_{21}$, where the constant value $H_{m e a n}^{\Gamma}$ was subtracted. Note that the colorbar is logarithmic.
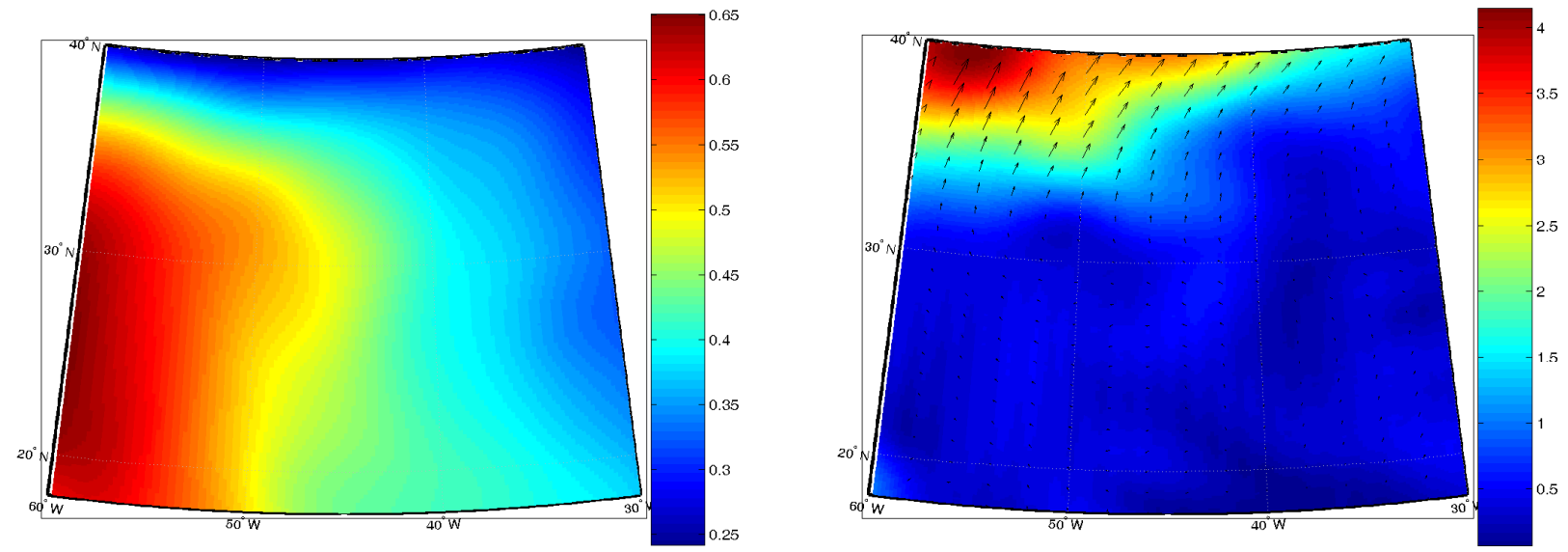

Fig. 7 Plot of the mean dynamic topography in [m] (left) and the geostrophic flow in $[\mathrm{cm} / \mathrm{s}]$ (right) in a rectangular region . 

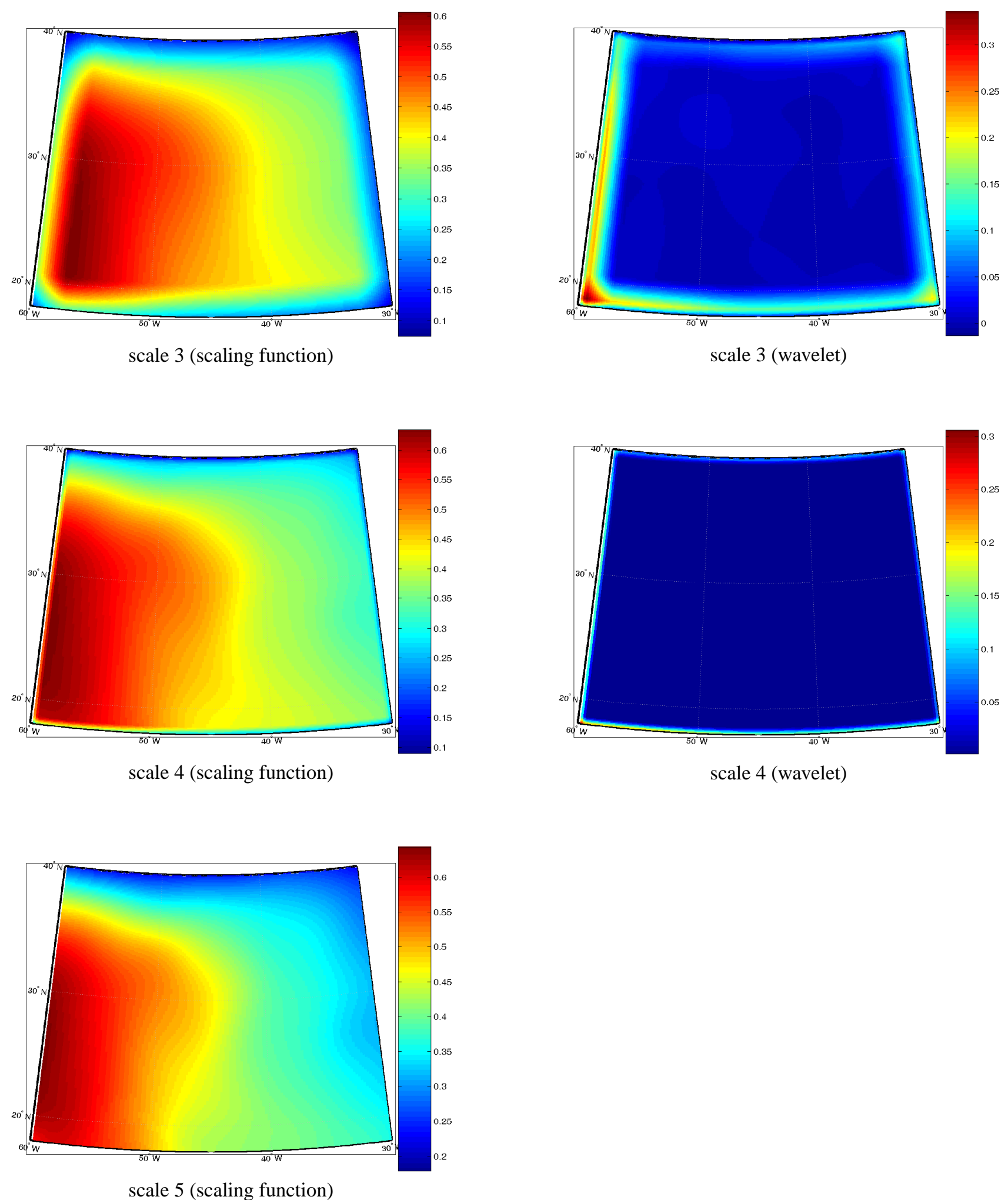

Fig. 8 Reconstruction of the topography in $[\mathrm{m}]$ on a rectangular region $\Gamma$ at low scales using the scale discretized regularized Green function. 


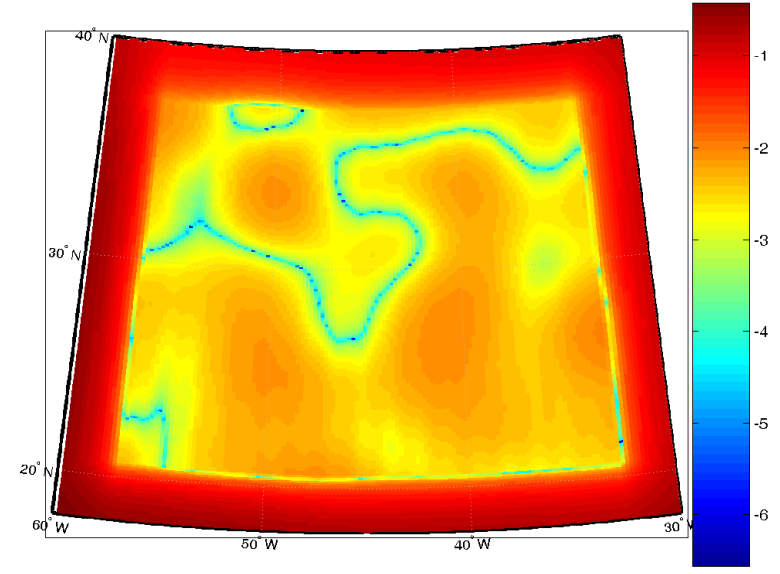

scale 3
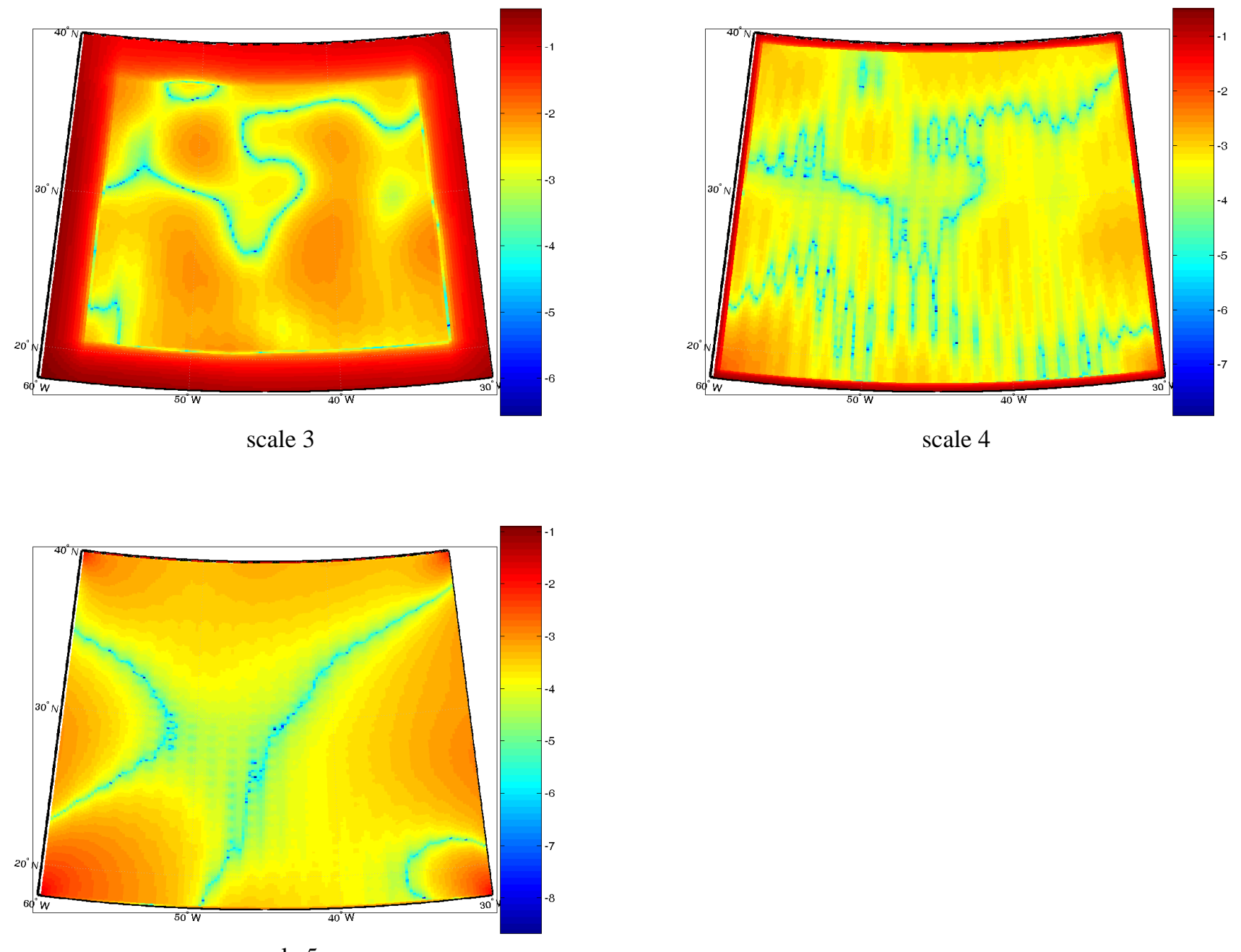

scale 5

Fig. 9 Approximation error of topography in a rectangular region $\Gamma$, where the constant value $H_{\text {mean }}^{\Gamma}$ was subtracted. Note that the colorbar is logarithmic. 

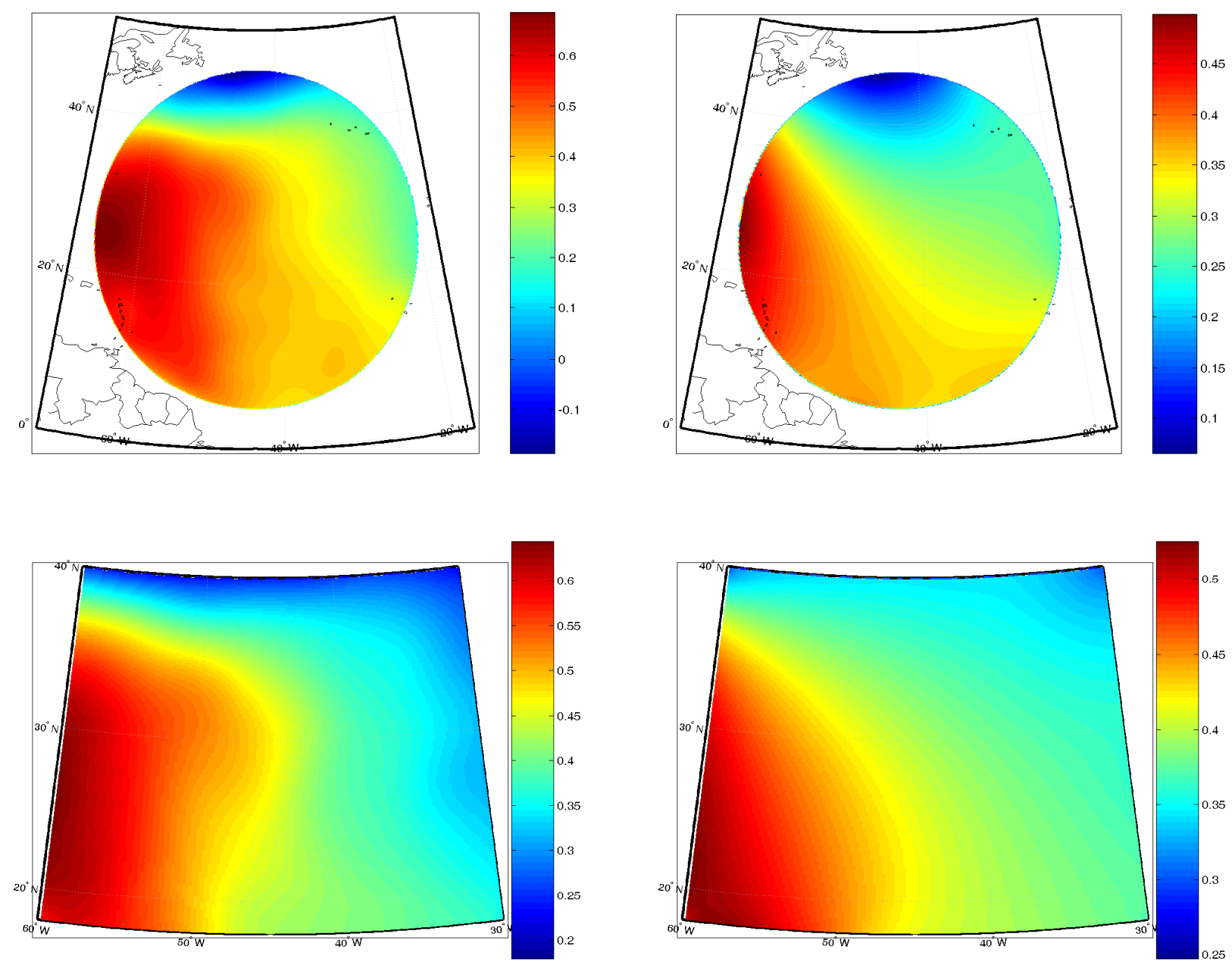

Fig. 10 Comparison of the total reconstruction of the dynamic topography in [m] (left) to the part of the reconstruction coming from the boundary integral (right) in the cap and in the rectangular region at scale 5. 
Folgende Berichte sind erschienen: 2003

Nr. 1 S. Pereverzev, E. Schock. On the adaptive selection of the parameter in regularization of ill-posed problems

Nr. 2 W. Freeden, M. Schreiner. Multiresolution Analysis by Spherical Up Functions

Nr. 3 F. Bauer, W. Freeden, M. Schreiner. A Tree Algorithm for Isotropic Finite Elements on the Sphere

Nr. 4 W. Freeden, V. Michel (eds.) Multiscale Modeling of CHAMP-Data

Nr. 5 C. Mayer Wavelet Modelling of the Spherical Inverse Source Problem with Application to Geomagnetism

\section{4}

Nr. 6 M.J. Fengler, W. Freeden, M. Gutting Darstellung des Gravitationsfeldes und seiner Funktionale mit Multiskalentechniken

Nr. 7 T. Maier Wavelet-Mie-Representations for Soleniodal Vector Fields with Applications to lonospheric Geomagnetic Data

Nr. 8 V. Michel Regularized Multiresolution Recovery of the Mass Density Distribution From Satellite Data of the Earth's Gravitational Field

Nr. 9 W. Freeden, V. Michel Wavelet Deformation Analysis for Spherical Bodies
Nr. 10 M. Gutting, D. Michel (eds.) Contributions of the Geomathematics Group, TU Kaiserlautern, to the 2nd International GOCE User Workshop at ESA-ESRIN Frascati, Italy

Nr. 11 M.J. Fengler, W. Freeden A Nonlinear Galerkin Scheme Involving Vector and Tensor Spherical Harmonics for Solving the Incompressible Navier-Stokes Equation on the Sphere

Nr. 12 W. Freeden, M. Schreiner Spaceborne Gravitational Field Determination by Means of Locally Supported Wavelets

Nr. 13 F. Bauer, S. Pereverzev Regularization without Preliminary Knowledge of Smoothness and Error Behavior

Nr. 14 W. Freeden, C. Mayer Multiscale Solution for the Molodensky Problem on Regular Telluroidal Surfaces

Nr. 15 W. Freeden, K. Hesse Spline modelling of geostrophic flow: theoretical and algorithmic aspects

\section{5}

Nr. 16 M.J. Fengler, D. Michel, V. Michel Harmonic Spline-Wavelets on the 3-dimensional Ball and their Application to the Reconstruction of the Earth's Density Distribution from Gravitational Data at Arbitrarily Shape Satellite Orbits

Nr. 17 F. Bauer

Split Operators for Oblique Boundary Value Problems 
Nr. 18 W. Freeden, M. Schreiner Local Multiscale Modelling of Geoidal Undulations from Deflections of the Vertical

Nr. 19 W. Freeden, D. Michel, V. Michel Local Multiscale Approximations of Geostrophic Flow: Theoretical Background and Aspects of Scientific Computing

Nr. 20 M.J. Fengler, W. Freeden, M. Gutting The Spherical Bernstein Wavelet

Nr. 21 M.J. Fengler, W. Freeden, A. Kohlhaas, V. Michel, T. Peters Wavelet Modelling of Regional and Temporal Variations of the Earth's Gravitational Potential Observed by GRACE

Nr. 22 W. Freeden, C. Mayer A Wavelet Approach to TimeHarmonic Maxwell's Equations

Nr. 23 M.J. Fengler, D. Michel, V. Michel Contributions of the Geomathematics Group to the GAMM $76^{\text {th }}$ Annual Meeting

Nr. 24 F. Bauer

Easy Differentiation and Integration of Homogeneous Harmonic Polynomials

Nr. 25 T. Raskop, M. Grothaus

On the Oblique Boundary Problem with a Stochastic Inhomogeneity

\section{6}

Nr. 26 P. Kammann, V. Michel Time-Dependent Cauchy-Navier Splines and their Application to Seismic Wave Front Propagation

Nr. 27 W. Freeden, M. Schreiner Biorthogonal Locally Supported Wavelets on the Sphere Based on Zonal Kernel Functions
Nr. 28 V. Michel, K. Wolf Numerical Aspects of a SplineBased Multiresolution Recovery of the Harmonic Mass Density out of Gravity Functionals

Nr. 29 V. Michel Fast Approximation on the 2Sphere by Optimally Localizing Approximate Identities

Nr. 30 M. Akram, V. Michel Locally Supported Approximate Identities on the Unit Ball

\section{7}

Nr. 31 T. Fehlinger, W. Freeden, S. Gramsch, C. Mayer, D. Michel, and M. Schreiner Local Modelling of Sea Surface Topography from (Geostrophic) Ocean Flow 


\section{TECHNISCHE UNIVERSITÄT
KAISERSLAUTERN}

Informationen:

Prof. Dr. W. Freeden

Prof. Dr. E. Schock

Fachbereich Mathematik

Technische Universität Kaiserslautern

Postfach 3049

D-67653 Kaiserslautern

E-Mail: freeden@mathematik.uni-kl.de schock@mathematik.uni-kl.de 